\title{
OBSERVATORIO
}

\section{INTELIGENCIA COMPETITIVA: LOGROS Y DESAFÍOS}

\author{
Alessandro Comai
}

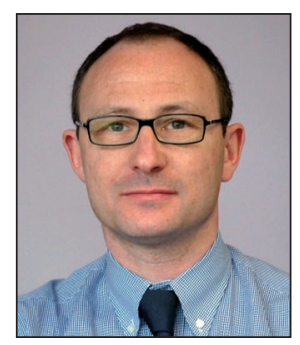

Alessandro Comai es ingeniero industrial (BSc Honor), MBA por la Universidad Pompeu Fabra (UPF), y DEA y doctorando en Ph. D. Management Science por Esade. Profesor asociado de la UPF. En 2005 fue profesor visitante en la Tampere University of Technology (Finlandia). Socio director de Miniera SL (http://www.miniera.es), consultora en IC cuyos clientes son organizaciones nacionales e internacionales, públicas y privadas (petróleo, química, industrial, consumo y servicios). Ideólogo del software para IC y VT Miralntelligence e IntelligenceSuite. Ha impartido cursos, seminarios y presentaciones en España (UPF, IDEC, IESE, TUT e ISM), EUA, Japón, Chile, Colombia, México, Brasil, Suiza, Alemania, Finlandia... Es co-director del primer curso de postgrado online en Inteligencia competitiva y vigilancia tecnológica (IDEC-UPF). Co-autor con Joaquín Tena-Millán de los libros Mapping \& anticipating the competitive landscape e Inteligencia competitiva y vigilancia tecnológica: experiencias de implantación en España y Latinoamérica; y co-autor con John E. Prescott del manual Establishing a world-class competitive intelligence function. Director de Puzzle - Revista hispana de la inteligencia competitiva. Miembro activo de la Society of Competitive Intelligence Professionals (SCIP) y del consejo directivo de Asepic.

Universitat Pompeu Fabra, Depto. de Economía y Empresa Edificio Jaume I, Ramon Trias Fargas, 25-27. 08005 Barcelona alessandro.comai@miniera.es; alessandro.comai@upf.edu http://www.miniera.es; http://www.econ.upf.edu

\section{Resumen}

Descripción de algunos de los temas críticos que un profesional de inteligencia competitiva y vigilancia tecnológica tiene que tener en cuenta a la hora de implantar tales funciones en una organización. Los logros y desafíos que se resumen en el texto provienen de la experiencia práctica del autor, tanto en la puesta en marcha de plataformas y unidades de inteligencia en instituciones gubernamentales y empresas españolas e internacionales, como de los trabajos de investigación realizados hasta el momento.

\section{Palabras clave}

Inteligencia competitiva, Necesidades, Puntos ciegos, Vigilancia tecnológica, Alerta temprana, Implantación de unidades de inteligencia, Señales débiles.

\section{Title: Competitive intelligence: successes and challenges}

\section{Abstract}

Description of some of the critical issues that a competitive intelligence and surveillance technology professional has to take into account when implementing such functions in an organization. The achievements and challenges summarized in the text come from the author's experience, both in the implementation of platforms and intelligence units in government and private companies in Spain and abroad, as well as from research work done so far.

\section{Keywords}

Competitive intelligence, Challenges, Needs, Blind spots, Technology monitoring, Early warning, Technology watch, Intelligence units implementation, Weak signals.

Comai, Alessandro. "Inteligencia competitiva: logros y desafíos". El profesional de la información, 2011, septiembreoctubre, v. 20 , n. 5 , pp. 489-493.

http://dx.doi.org/10.3145/epi.2011.sep.01

\section{Introducción}

Hace más de 13 años empecé a trabajar en inteligencia competitiva con mi amigo y socio Joaquín Tena para culminar en nuestro proyecto empresarial que se llama Miniera. En aquel entonces se hablaba casi exclusivamente de "vigilancia tecnológica" y el término "inteligencia competitiva" se conocía escasamente en España ${ }^{1}$ y países latinoamericanos. 
La anécdota es que en 1999, cuando estuvimos recopilando fuentes de información disponibles en internet para crear un CD y ofrecerlo a los empresarios, Joaquín y yo hablábamos de llamarlo "Información competitiva" ya que "inteligencia" podría tener diferentes interpretaciones. Ya conocemos todos cómo el término ha ido evolucionando a lo largo de los años y cómo en 2008 casi se había quintuplicado el número de apariciones de inteligencia competitiva en relación con vigilancia tecnológica que se pueden contar en la bibliografía (figura 1).

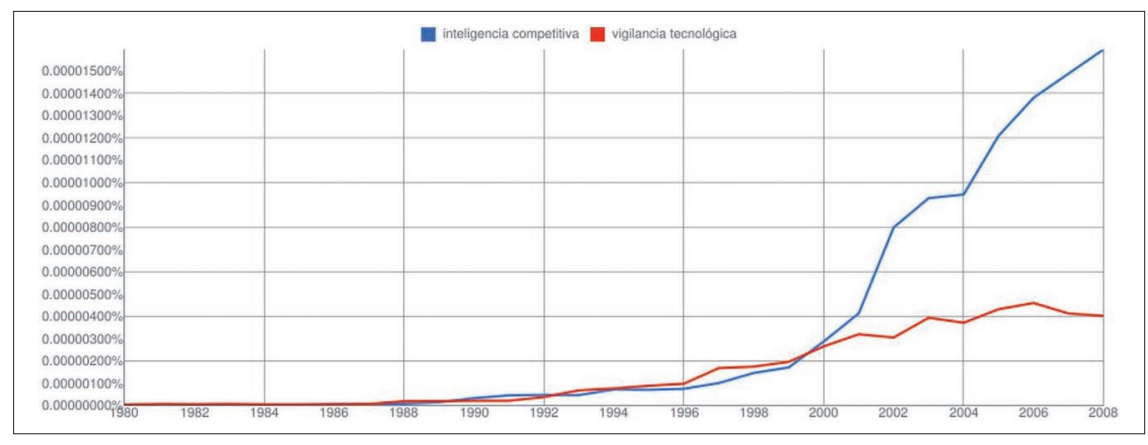

Figura 1. Evolución del uso de los términos "inteligencia competitiva" y "vigilancia tecnológica" en libros en español según Books Ngram Viewer de Google http://bit.ly/nrYbEI

http://ngrams.googlelabs.com/graph?content=inteligencia+competitiva, vigilancia+tecnológica\&yea r_start $=1980 \&$ corpus $=10$

\section{Difusión de la disciplina}

Creo que hay diferentes razones que justifican el éxito que ha tenido la difusión de la IC. Una de ellas es que tiene un sentido más amplio, lo que facilita que más empresas, directivos y departamentos dentro de la empresa comprendan pronto que les afecta y puede serles útil. En efecto, todas las empresas y organizaciones pueden aplicarla al campo comercial, competitivo, tecnológico, normativo, económico, geopolítico, y medioambiental, entre otros.

Otro aspecto que ha favorecido la difusión de la IC han sido las publicaciones y cursos que la han promocionado. Por ejemplo, en 2002 fundamos Puzzle, revista de inteligencia competitiva, que publicó 32 números sobre la IC y la VT en

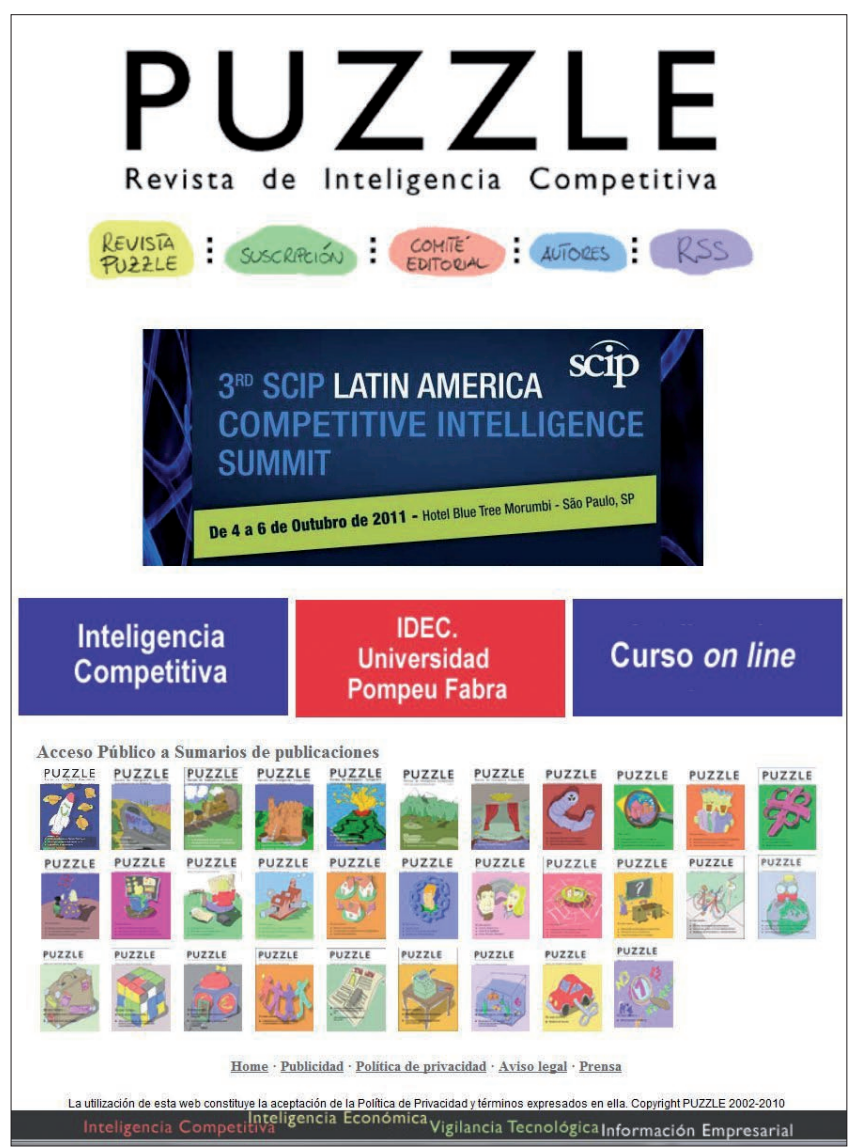

Todavía pueden leerse todos los números de la revista Puzzle http://www.revista-puzzle.com/index.php todos sus aspectos, y llegó a tener más de 4.000 suscriptores de diferentes sectores, organizaciones y especializaciones.

El otro grano de arena que ha contribuido a esta difusión ha sido el primer Curso online de inteligencia competitiva que lanzamos en la Universidad Pompeu Fabra y que hoy ha llegado a su 13a edición².

Sin embargo, la IC en España se ha enfrentado a importantes desafíos para su implantación. Ha tenido lugar una lenta y gradual penetración en la empresa privada y sólo se ha introducido excepcionalmente en el curriculum de los estudios de administración de empresa. Cabe destacar el importante esfuerzo realizado por la Asociación Española para la Promoción de la Inteligencia Competitiva (Asepic) en estos últimos años en difundir y promover la IC en todos los sectores, ámbitos territoriales y organizaciones.

http://www.asepic.es

La situación económica actual no parece favorecer la inversión en IC, lo cual es un craso error, pues es ahora cuando las empresas necesitan la mejor información de que puedan disponer. Corren tiempos de crisis, y algunos vaticinan incluso un cambio fundamental de los modelos de crecimiento económico. Mi sensación es que se comprende mejor la necesidad de la inteligencia competitiva en algunos países emergentes y en el ámbito público que en las empresas privadas locales. Es sorprendente, teniendo en cuenta las dificultades crecientes para decidir dónde invertir, adaptarse, ampliar o reducir para poder afrontar los nuevos retos organizacionales, regionales y nacionales.

Muchos tenemos la convicción de que la crisis sólo se superará mediante la innovación y el cambio, en todos los ámbitos. Si eso es así, la necesidad de comprender el entorno y anticipar la evolución futura tanto de los sectores económicos como de los competidores es ahora más acuciante que nunca. Debiera adquirir más relieve la línea argumental que va desde los datos a la información, al conocimiento y, finalmente, a las decisiones acertadas. La identificación de nuevas fuentes de competitividad es ahora, si cabe, de mayor prioridad tanto para los gobiernos como para las empresas.

Esa "línea argumental" se sustenta en el trabajo de investigación y análisis previo a la toma de decisiones que caracteriza a la IC. Resulta paradójico que cuando más lo necesitan menos dispuestas estén las organizaciones a realizar los cambios y efectuar las inversiones para desarrollar un 


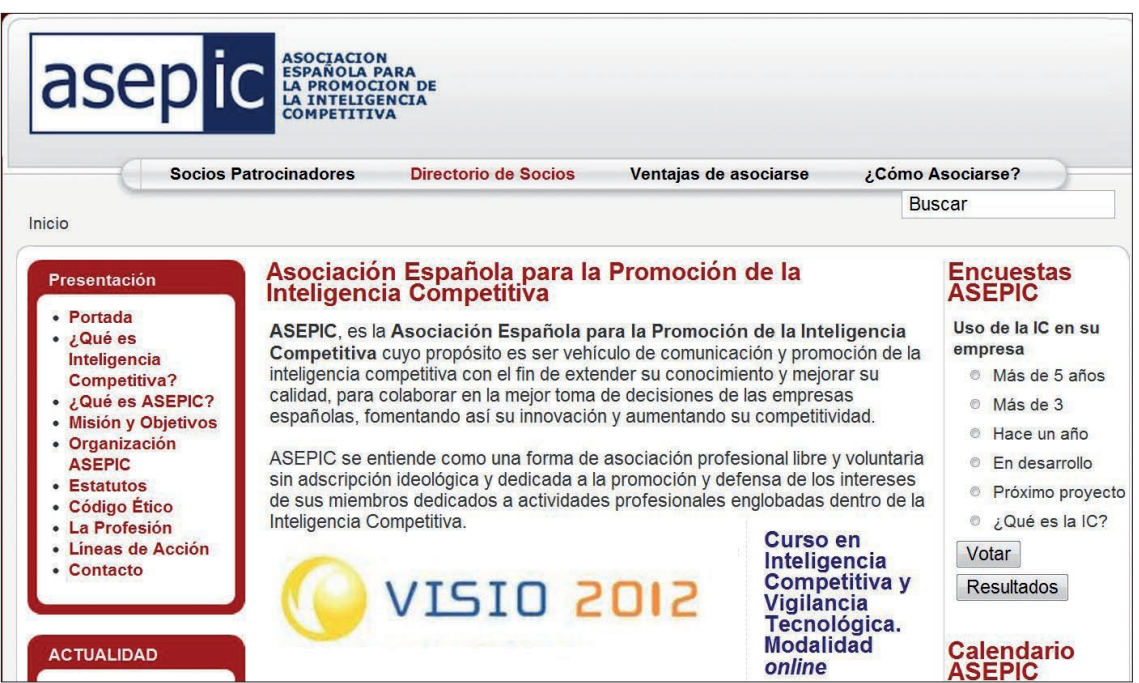

http://www.asepic.es

recurso (la IC) que facilitaría, de estar disponible y en plena madurez operativa, la adaptación a un entorno nuevo.

Resulta paradójico que ahora cuando más lo necesitan las organizaciones estén menos dispuestas a realizar inversiones en IC, lo que les facilitaría adaptarse al nuevo entorno

A pesar de las dificultades, existen empresas exitosas que utilizan la IC como una verdadera fuente de sabiduría para apoyar sus decisiones. Estas empresas no tienen porque ser grandes ni tampoco dedicar enormes recursos a la IC. Se trata de una cultura empresarial-que incorpora las ideas y alienta los comportamientos adecuados- lo que guía estas empresas a perseverar en la innovación y lograr sus objetivos.

\section{Cadena de valor en la inteligencia}

La puesta en marcha de una función de IC en una organización, sea privada o pública, empieza por una buena comprensión tanto de las oportunidades que puede brindar como de sus limitaciones. Es un desafío importante ya que hay que evitar falsas expectativas sobre sus ventajas, y sopesar los costes, el alcance y el valor estratégico que puede tener.

Es necesario: 1) una buena elección de las fuentes de información, 2) su tratamiento e interpretación, así como 3) un estudio sobre cómo se tiene que aplicar el conocimiento adquirido para obtener el máximo provecho de cualquier indicio o novedad que se produzca en el entorno. Los tres pasos son fundamentales para poder provocar un cambio de dirección tanto estratégica como táctica de la organización. En cierto modo estas tres etapas representan la cadena de valor de la in-

http://www.scip.org teligencia, pero no por conocidas deja de tener su importancia su ejecución con el mejor nivel posible de profesionalidad.

Por experiencia sabemos que la gran mayoría de las actividades de inteligencia llegan a crear muy buenos flujos de información pero lamentablemente se frenan ya en la primera etapa. Un ejemplo evidente es la proliferación de observatorios, que como dice la palabra, observan o vigilan el entorno, pero en general se limitan a ello. Crear un observatorio no requiere grandes esfuerzos conceptuales y metodológicos aunque puede requerir una inversión económica de cierta importancia. En España en concreto se han visto numerosos proyectos de portales que no han llegado a añadir el valor esperado por parte de los grupos de interés que representan a los usuarios finales, entre ellos las empresas.

Una parte importante de los recursos se tendría que dedicar a la formación y capacitación de los analistas y especialistas con el fin de que estén preparados para identificar e interpretar nuevos cambios, fenómenos y acontecimientos, y aportar consideraciones útiles para la toma de decisiones. Es decir, habría que intensificar la atención que se presta al desarrollo de las etapas de análisis y de utilización de su producto, la inteligencia. Normalmente la formación está contemplada en los proyectos de implantación y desarrollo de una función de IC, pero puede no recibir la prioridad adecuada al comienzo del proyecto, de modo que sufra retrasos e insuficiente dotación presupuestaria por haberse gastado la gran mayoría de recursos en las etapas previas. Una buena y meticulosa gestión del proyecto es clave para poder llevar a cabo de forma equilibrada las actividades de IC.

\section{Evolución de la profesión y sus instrumentos}

Los analistas de IC en ejercicio tienen que seguir formándose en las técnicas, modelos y métodos de la profesión. Así, por ejemplo, los artículos de inteligencia competitiva que

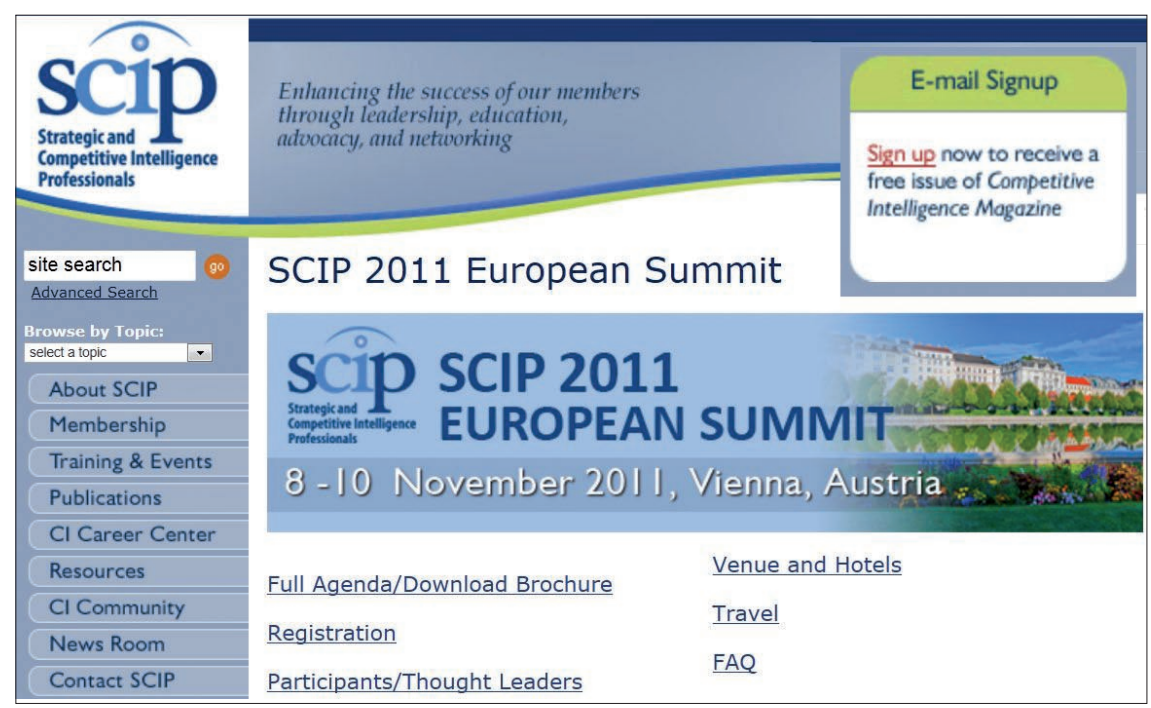


publica esta revista El profesional de la información en este número favorecen la formación continua de los profesionales. Quizá habría que reflexionar sobre el alto porcentaje de artículos rechazados que ha habido, según me informan los editores de $E P I$, que quizá sería indicativo de todavía cierta falta de madurez de esta profesión.

Una de los temas que emergen con fuerza y que han obligado a una nueva orientación de la práctica de la IC es la "inteligencia online". Esta especialidad requiere un buen conocimiento del funcionamiento de las redes sociales, de los buscadores y el posicionamiento de la marca, de la web profunda, de las bases de datos..., entre otros aspectos.

Cadena de valor de la IC: buenas fuentes de información, correcta interpretación y aplicación adecuada

Para poder desempeñar esta actividad es necesario conocer herramientas de data mining que permiten analizar grandes cantidades de datos disponibles en internet. Existen softwares gratuitos o de bajo coste que permiten acelerar el ciclo de inteligencia, aunque han sido diseñados para el uso individual más que corporativo. En una encuesta ${ }^{3}$ que realizamos durante mayo-junio de 2011 se analizaron más de 24 herramientas libres para la IC, pero se demostró que los profesionales hacen un uso limitado de ellas y que lo más utilizado es el buscador de Google.

Los resultados de dicha encuesta muestran una fotografía parcial de las actividades de los profesionales de IC dado el limitado número de herramientas libres que fueron citadas. En internet existen centenares de herramientas que se pueden utilizar para capturar, filtrar, categorizar, analizar, visualizar y distribuir la información en diferentes formatos. Desconocemos tanto el número de proyectos continuos o puntuales que los profesionales encuestados ejecutan en su actividad, como la intensidad de uso de las herramientas dedicadas a ellos. Sin embargo el hecho de declarar que usan Google como aplicación principal nos hace pensar que el desempeño mayor de los profesionales de IC está concentrado en actividades puntuales. Y si fuera así, difícilmente lograrán anticiparse en el entorno que les rodea.

\section{Dos enfoques y elementos en la alerta temprana}

Existen adicionalmente otras actividades que los profesionales de IC tienen que tener en cuenta. Una de ellas es la capacidad de anticipar los acontecimientos. En relación con este aspecto se pueden crear sistemas de alerta temprana. Reúnen un conjunto de instrumentos que pueden ser empleados tanto para apoyar el logro de los retos (objetivos) de una organización, como para identificar cambios del entorno, tendencias incipientes u otros fenómenos que podrían tener implicaciones importantes para la organización.

La alerta temprana necesita dos elementos clave para poder detectar las señales débiles de cambio:

1) un flujo constante y exhaustivo de información y/o datos provenientes de múltiples fuentes, entre ellas las no estrictamente pertenecientes al propio sector de actividad, y

2) un proceso abierto, flexible y adaptable de interpretación de la información y los datos que permita reconocer los cambios en las señales.

Tanto uno como otro elemento son indispensables para poder estructurar un sistema de anticipación.

Hace algunos años Joaquín Tena y yo escribimos un libro titulado Mapping \& anticipating the competitive landsca$p e^{4}$ que abordaba el tema de la anticipación del entorno en el marco de la IC. La obra describe un modelo donde los acontecimientos o supuestos previamente identificados se analizan en base a un grupo de indicadores entrelazados. Este modelo ha sido utilizado en empresas petroleras, financieras, etc., para realizar el seguimiento de unos temas determinados. Definimos nuestro modelo como una de las herramientas que apoyan los sistemas de alerta "focalizados", sin embargo tales sistemas no resuelven el problema de anticipar los acontecimientos que no percibimos o no hemos supuesto previamente. Es decir, no ayudan a identificar los inesperados nuevos acontecimientos que emergen tenuemente en el entorno y que pueden transformarse en señales débiles de cambios, quizá importantes en el futuro. Eso nos condujo a concluir que es necesario adoptar además un sistema de alerta temprana "no focalizada" y que justamente necesita los dos condicionantes descritos anteriormente.

Los dos modelos no son mutuamente excluyentes ya que por un lado el no focalizado permite identificar acontecimientos, y por el otro el focalizado realizar su seguimiento para trasformarlos en señales. En ambos casos el reto mayor de cualquier organización es ampliar la capacidad de interpretación de la información, ya que ésta pone en discusión los supuestos básicos sobre los cuales los directivos fundamentan sus decisiones.

\section{El mayor reto de cualquier organización es interpretar la información obtenida y aplicar los cambios, ya que ello implica poner en discusión los supuestos bási- cos sobre los cuales los directivos funda- mentan sus decisiones}

Entendemos que la puesta en marcha de este proceso no es fácil ya que requiere un esfuerzo por parte de la organización, que incluye dedicar recursos humanos, tecnológicos y financieros. Asimismo la organización misma necesita implicarse en estas actividades, ya que como comentábamos, la interpretación multidisciplinar de la información puede y debe ser ejecutada por parte de toda la organización.

Este aspecto puede ser resuelto solamente cuando se logra desarrollar una cultura organizacional orientada a la IC, que seguramente es la parte más difícil de la introducción de la IC en una organización. El resultado de los perfiles de más de 200 empresas que han utilizado el modelo de autoevaluación ${ }^{5}$ que hemos desarrollado a partir de 2003 demues- 


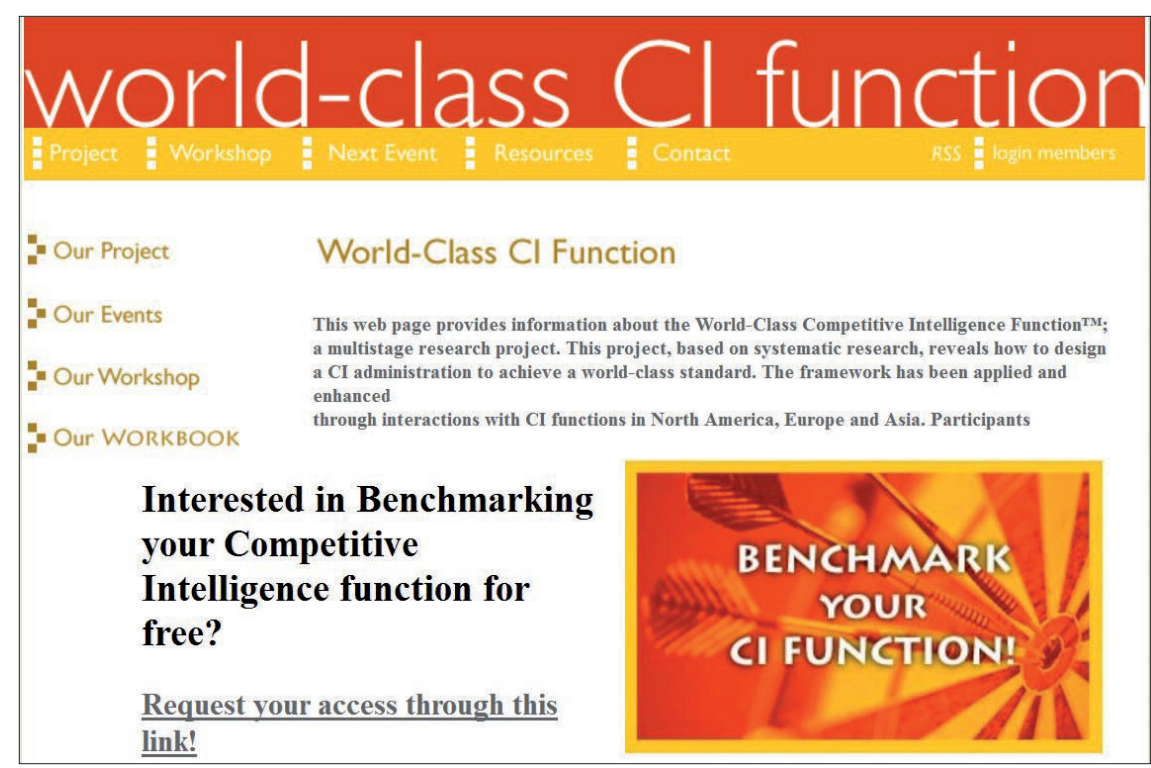

World class $\mathrm{Cl}$ function ${ }^{T M}$ es un proyecto que ha analizado de forma sistemática la implementación de unidades de IC en empresas y organizaciones. En su web se explica cómo crearlas de forma óptima. http://www.world-class-ci.com

tra que tanto la cultura de capturar como la de compartir inteligencia es la menos desarrollada por las empresas. Nuevamente nos encontramos con que la IC necesita una muy importante gestión del cambio cultural por parte de la organización y esto sin duda supone un cierto tiempo y perseverancia.

\section{Conclusión}

Con la ayuda de la IC y con el tiempo y la dedicación adecuados es posible superar los retos que hemos descrito. Tarde o temprano las empresas de éxito se dan cuenta de las importantes ventajas que se obtienen identificando las innovaciones disruptivas de su sector, y cambiando oportunamente el modelo de negocio o rompiendo con los supuestos tradicionales de gestión de la empresa o de la industria.

Si evaluar el precio de la información es difícil, el de la no-información es casi imposible

Por desgracia, la experiencia de las organizaciones fracasadas o con resultados pobres por no haberse anticipado a los cambios son difíciles de transmitir para que sirvan de ejemde consultar en: plo. Si evaluar el precio de la información es difícil, el de la no-información es casi imposible, con el agravante de que los que sufren las consecuencias de su ignorancia no acostumbran a darse cuenta de que el desastre se podía haber prevenido.

\section{Notas}

1. Fue la traducción del término inglés competitive intelligence usado por la sociedad Strategic and Competitive Intelligence Professionals (SCIP), -llamada Society of Competitive Intelligence Professionals hasta julio de 2010-.

2. El programa del curso Cicol puede verse en:

http://www.idec.upf.edu/curso-eninteligencia-competitiva-y-vigilanciatecnologica

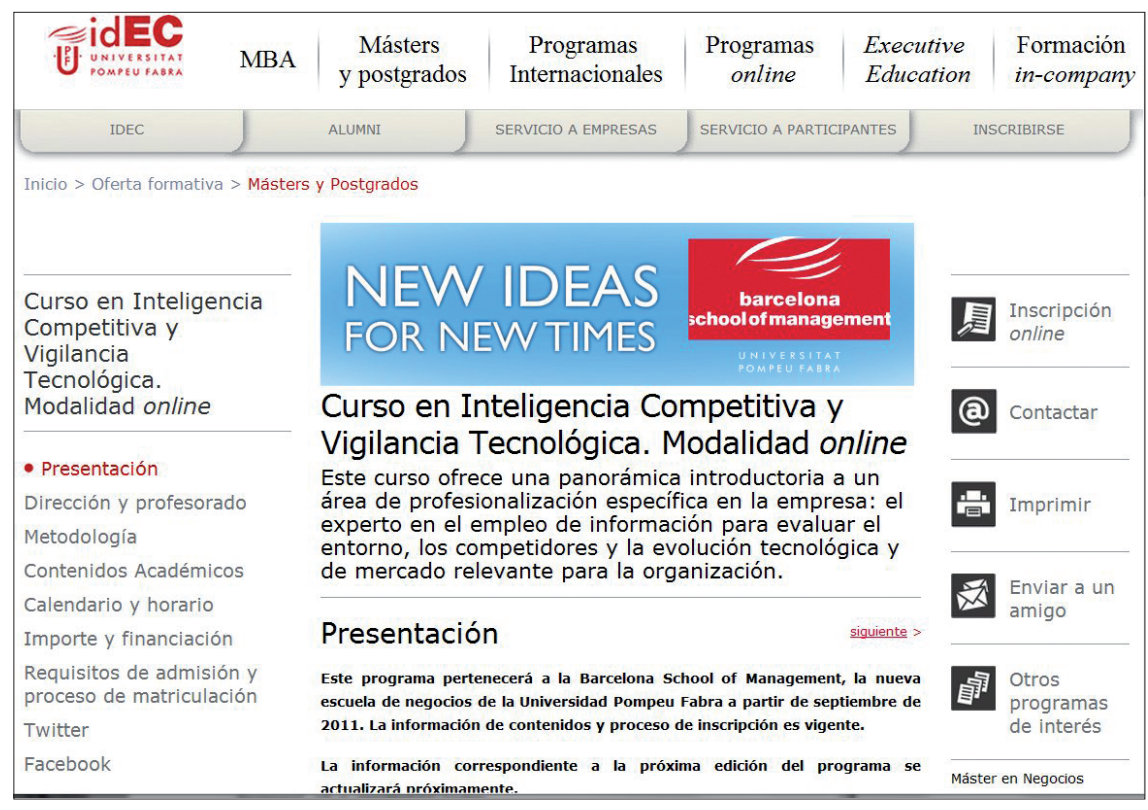

http://www.idec.upf.edu/curso-en-inteligencia-competitiva-y-vigilancia-tecnologica

3. La presentación de los resultados de la encuesta "Google Tools for Cl", disponible solamente en idioma inglés, se pue-

http://www.miniera.es/es_resultados_articles.htm

4. El libro "Mapping \& anticipating the competitive landscape", publicado en 2006, puede ser consultado en: http://www.miniera.es/es_resultados_articles.htm

5. Al modelo de autoevaluación "World class $\mathrm{Cl}$ function ${ }^{\mathrm{TM}}$ " se puede acceder a través de la web:

http://www.world-class-ci.com 


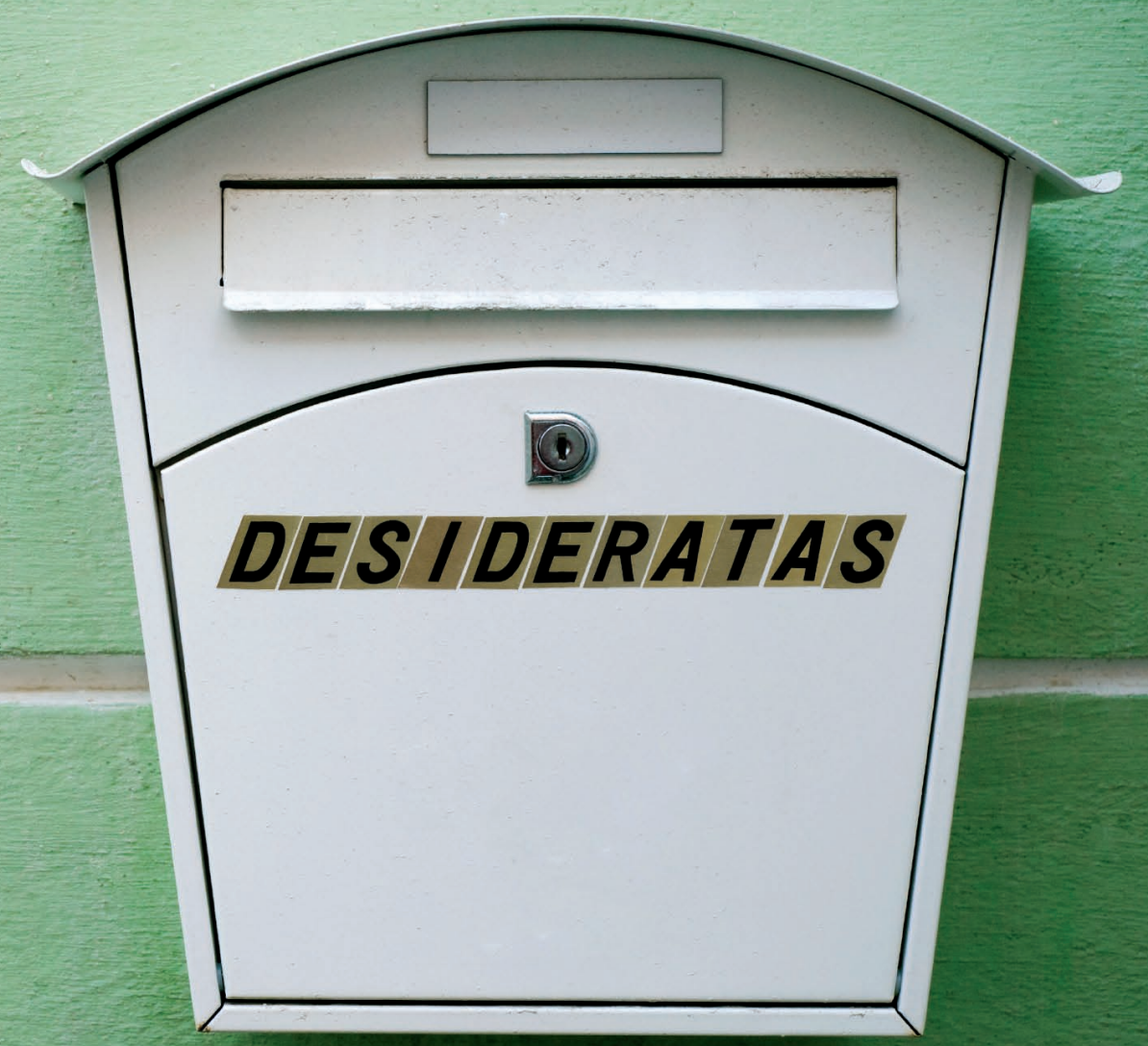

\section{Te entendemos 99}

Sistemas de gestión de bibliotecas Open Source Interfaces interactivas y OPACs Repositorios OAI

Gestión documental y de archivos

Digitalización

Outsourcing de servicios documentales

Desarrollo de sitios web / multimedia / e-learning

Comunicación y e-marketing de servicios de información
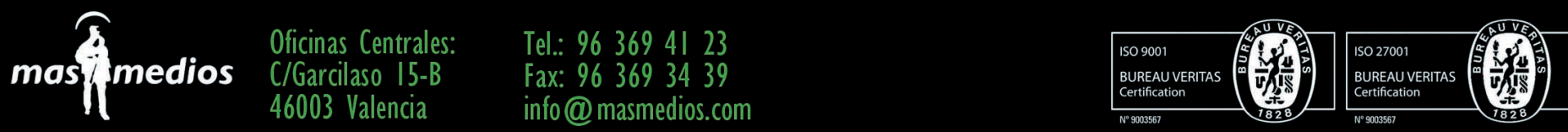